\title{
4-PAM for High-Speed Short-Range Optical Communications
}

\author{
Krzysztof Szczerba, Petter Westbergh, Johnny Karout, Johan S. Gustavsson, Åsa Haglund, \\ Magnus Karlsson, Peter A. Andrekson, Erik Agrell, and Anders Larsson
}

\begin{abstract}
In this work, we compare 4-pulse amplitude modulation and on-off keying modulation formats at high speed for short-range optical communication systems. The transmission system comprised a directly modulated verticalcavity surface-emitting laser operating at a wavelength of $850 \mathrm{~nm}$, an OM3 + multimode fiber link, and a photodetector detecting the intensity at the receiver end. The modulation formats were compared both at the same bit-rate and at the same symbol rate. The maximum bit-rate used was 25 Gbps. Propagation distances up to $600 \mathrm{~m}$ were investigated at 12.5 Gbps. All measurements were done in real time and without any equalization.
\end{abstract}

Index Terms-Data communications; Fiber-optical communications; IM/DD; Interconnects; MMF; 850 nm; OOK; 4-PAM; Short range; VCSEL.

\section{INTRODUCTION}

$\mathbf{T}$ he increasing demand for capacity in short-range optical communications, such as optical interconnects and storage area networks, has motivated the development of fast and low-cost vertical-cavity surface-emitting lasers (VCSELs) and multimode fiber (MMF). Graded index MMF, optimized for the $850 \mathrm{~nm}$ wavelength, has been successfully used to reduce the impact of modal dispersion induced intersymbol interference (ISI) and extend the reach in such systems. Currently, 10 Gbps links are commercialized, and lasers and photoreceivers (although with limiting amplifiers only) for $25 \mathrm{Gbps}$ at the wavelength of $850 \mathrm{~nm}$ are becoming available. However, with increased bit-rates and the recent development of VCSELs capable of operating at $40 \mathrm{Gbps}$ at the wavelength of $850 \mathrm{~nm}$ [1] and $44 \mathrm{Gbps}$ at the wavelength of $980 \mathrm{~nm}$ [2], it turns out that the transmission distance in MMF at such high bit-rates is limited by the modal dispersion, if on-off keying (OOK) modulation is used. Multilevel modulation, with higher spectral efficiency, is a potential way to extend the reach of MMF-based links at high bit-rates. Because of cost constraints, intensity modulation and direct detection (IM/DD) is appealing in short-range optical networks.

There are two main possibilities for increasing the spectral efficiency in IM/DD links: subcarrier modulation (SCM) and pulse amplitude modulation (PAM). In SCM schemes, a

Manuscript received April 20, 2012; revised August 25, 2012; accepted September 7, 2012; published October 31, 2012 (Doc. ID 167141).

The authors are with the Department of Microtechnology and Nanoscience, Chalmers University of Technology, Göteborg, Sweden (e-mail: krzysztof. szczerba@chalmers.se).

Digital Object Identifier 10.1364/JOCN.4.000885 microwave subcarrier signal is first modulated with, for example, quadrature amplitude modulation (QAM) or phase shift keying modulation (PSK). Single-cycle SCM with 16-QAM has been demonstrated in links using VCSELs and MMFs [3], with the transmitter operating in real time and the digital processing on the receiver side being implemented off-line. Discrete multi-tone modulation, which is a multiple subcarrier scheme, has been demonstrated at $30 \mathrm{Gbps}$ for the same type of link [4], also with off-line processing. The main advantages of the multiple subcarrier approaches are easier electronic equalization and increased robustness to effects of modal dispersion due to reduced symbol rates. A general disadvantage of IM/DD subcarrier schemes, compared to PAM, is worse sensitivity, in terms of optical received power [5,6]. Another disadvantage of the subcarrier schemes is their implementation complexity, whether implemented using analog electronics or digital signal processing.

The complexity of the electronic modulator, demodulator and laser driver circuits is an important limitation in the design of short-range data communication links. Higher complexity means higher power consumption, which is undesired in densely packed data centers, and therefore it is difficult to justify use of subcarrier modulation in such environments. On the other hand, PAM offers probably the lowest implementation complexity of all multilevel modulation formats. Complementary metal-oxide-semiconductor (CMOS) electronic circuits for real-time 4-PAM transmitters and receivers, operating at bit-rates up to $22 \mathrm{Gbps}$, have already been developed [7,8]. In [9], multilevel intensity modulation formats, including PAM in particular, were investigated for increasing the reach of $10 \mathrm{Gbps}$ links, using $1550 \mathrm{~nm}$ wavelength and standard single-mode fiber. It was shown that 4-PAM modulation can increase the dispersion-limited distance. Electronic pre-distortion for extension of the reach of 4-PAM in MMF was analyzed and demonstrated at $10 \mathrm{Gbps}$ in [10], and eye diagrams from real-time operation at $32 \mathrm{Gbps}$ with electronic pre-distortion were demonstrated in [11]. In [12], 4-PAM and OOK were compared in short-range optical links with VCSELs and MMF, with promising results.

Recently, we have demonstrated 30 Gbps error-free transmission over $200 \mathrm{~m}$ of MMF, using 4-PAM and a $850 \mathrm{~nm}$ VCSEL [13]. The 4-PAM signal was generated in real time and the bit error rate (BER) measurement was also done in real time. No equalization was used in the receiver, nor was any pre-distortion used in the transmitter. Although electronic equalization may become commonplace in the future, implemented for example with transversal filters [11], the objective of this work is to provide a baseline comparison 
of OOK and 4-PAM. A detailed description of the experimental procedures was presented in [14].

In this work, we further investigate the application of 4-PAM in short-range optical links using VCSELs, MMF, and direct detection. We present theoretical and experimental comparisons of the sensitivity and ISI penalties of 4-PAM and OOK. An experimental comparison of sensitivities and ISI penalties for both modulation formats is also presented.

There are two cases of particular interest when comparing OOK and 4-PAM. The first one is when 4-PAM is used to double the bit-rate, compared to an OOK system. The main questions are how much more optical power is required and how the propagation in the MMF is affected. To answer these questions we have compared experimentally OOK and 4-PAM at the same symbol rate of 12.5 Gbaud, which in other words meant comparison of 12.5 Gbps OOK with 25 Gbps 4-PAM.

The other case is when 4-PAM is run at the same bit-rate as OOK, to increase the propagation distance in MMF. The main interesting question here is how large the improvement in propagation distance in the MMF is, and again, what the power penalty is. From a simplistic point of view, the propagation distance should be doubled, because of the signal bandwidth being reduced by half, but, on the other hand, the power expense for using more levels means that less dispersion penalty can be tolerated. To investigate this, we have experimentally compared 4-PAM and OOK at $12.5 \mathrm{Gbps}$.

This paper is organized as follows. In Section II, we briefly compare OOK and 4-PAM theoretically. In Section III, we elaborate on the experimental setup. In Section IV, we present experimental results and discussion. Section V contains closing conclusions.

\section{4-PAM AND OOK FOR SHORT-RANGE LINKS-THEORETICAL CONSIDERATIONS OF BER PERFORMANCE AND SENSITIVITY}

\section{A. Relative Sensitivity}

With the limited bandwidth-distance product of MMFs it is desirable to reduce the signal bandwidth. Unfortunately, with OOK modulation this means that the data rate will also be reduced. However, if multilevel modulation, such as PAM, is used, the bandwidth of the signal can be reduced, for the same bit-rate, at the expense of the optical power efficiency, manifested through the receiver sensitivity. The bandwidth of $M$-level PAM is $1 / \log _{2}(M)$ of the OOK signal bandwidth at the same bit-rate, where $M$ is the number of levels [9]. For 4-PAM, this means that the bandwidth is reduced by half, compared to OOK at the same bit-rate. Under assumptions that the noise is additive, white, and stationary, the optical power penalty for using a multilevel PAM, compared to OOK at the same symbol rate, in terms of the required optical power in $\mathrm{dB}$ to reach the same BER is

$$
P_{p s}=10 \log _{10}(M-1)
$$

where $M$ is the number of PAM levels [15]. This means that $4.8 \mathrm{~dB}$ more received optical power is needed for 4-PAM at the same symbol rate as OOK. The penalty is less when the bit-rate is kept fixed, because of reduced bandwidth. The optical power penalty for $M$-PAM, relative to OOK at the same bit-rate $P_{p b}$, is expressed in $\mathrm{dB}$ as

$$
P_{p b}=10 \log _{10}\left(\frac{M-1}{\sqrt{\log _{2}(M)}}\right),
$$

according to [9]. For 4-PAM this gives that $3.3 \mathrm{~dB}$ more optical power is required compared to the OOK signal at the same bit-rate to reach the same BER.

\section{B. SER and BER Calculation}

To calculate the theoretical BER values for $M$-PAM systems, we start with the symbol error rate (SER) calculation. Assuming that all $M$ symbols are equiprobable, the SER can be calculated in an exhaustive manner through

$$
\mathrm{SER}=\frac{1}{M} \sum_{i=0}^{M-1} \sum_{j=0, j \neq i}^{M-1} P_{i j},
$$

where $P_{i j}$ is the probability of receiving symbol $j$ when symbol $i$ was transmitted [16, Ch. 4.2]. Assuming Gaussian noise, this probability is found as

$$
P_{i j}=\frac{1}{2} \operatorname{erfc}\left(\frac{I_{t h, j}-I_{i}}{\sigma_{i} \sqrt{2}}\right)-\frac{1}{2} \operatorname{erfc}\left(\frac{I_{t h, j+1}-I_{i}}{\sigma_{i} \sqrt{2}}\right) .
$$

Here, $I_{i}$ denotes the photocurrent at symbol $i$, and $I_{t h, j}$ is the threshold current, where $I_{t h, 0}=-\infty$, and $I_{t h, M}=+\infty$. The remaining decision thresholds are located between the subsequent symbols. The root mean square (RMS) value of the noise current at the symbol $i$ level is denoted $\sigma_{i}$.

The BER is dependent on the labeling of the symbols. The Gray labeling usually provides the best performance [17], but sometimes in experiments it is easier to implement natural labeling. In general, the BER can be expressed as

$$
\mathrm{BER}=\frac{1}{M} \sum_{i=0}^{M-1} \sum_{j=0, j \neq i}^{M-1} \frac{d_{i j}}{\log _{2}(M)} P_{i j},
$$

where the $d_{i j}$ is the Hamming distance between the labels of symbols $i$ and $j$ [17]. An example of the two labelings for $M=4$ is given in Table I.

Assuming that the thermal noise is dominating, all symbol levels are equally spaced, and the decision thresholds are equidistant from adjacent symbols, the SER can be expressed as

$$
\mathrm{SER}=\frac{M-1}{M} \operatorname{erfc}\left(\frac{I_{\mathrm{avg}}}{(M-1) \sqrt{2} \sigma}\right),
$$

where $I_{\mathrm{avg}}$ is the average photodetector current and $\sigma=\sigma_{i}$ for all $i$ is the RMS noise current. For high signal to noise ratios (SNRs), it can be assumed that only errors between adjacent symbols occur. In that case, the BER can be approximated as

$$
\mathrm{BER}_{\text {approx }} \approx d_{\mathrm{avg}} \frac{\mathrm{SER}}{\log _{2}(M)},
$$


TABLE I

GRAY AND NATURAL LABELING FOR $M=4$

\begin{tabular}{lll}
\hline Symbol & Gray labeling & Natural labeling \\
\hline 3 & 10 & 11 \\
\hline 2 & 11 & 10 \\
\hline 1 & 01 & 01 \\
\hline 0 & 00 & 00 \\
\hline
\end{tabular}

where $d_{\text {avg }}$ is the average Hamming distance between the labels of adjacent symbols. If Gray labeling is used, $d_{\mathrm{avg}}=1$, which yields

$$
\mathrm{BER}_{G} \approx \frac{\mathrm{SER}}{\log _{2}(M)}
$$

If natural labeling is used, the BER is still well approximated by Eq. (7), where in this case

$$
d_{\mathrm{avg}}=\frac{\sum_{k=0}^{\log _{2}(M)-1}\left(\log _{2}(M)-k\right) 2^{k}}{M-1}=2-\frac{\log _{2}(M)}{M-1} .
$$

\section{Theoretical BER and Sensitivity for Our Experimental System}

The value of $I_{i}$ is related to the optical power $P$ as $I_{p}=R P$, where $R$ is the responsivity of the photodetector. The total noise current variance can be expressed as

$$
\sigma_{k}^{2}=4 k_{B} T F_{n} \Delta f / R_{L}+2 q I_{i} \Delta f+R I N I_{i}^{2} \Delta f,
$$

where the three terms of the sum on the right-hand side of the equation describe, respectively, the thermal noise, the shot noise, and the relative intensity noise (RIN) [18]. The values denoted by $k_{B}, T, F_{n}, \Delta f, R_{L}, q$, and RIN are, respectively, the Boltzmann constant, temperature in Kelvin, receiver amplifier noise figure, receiver bandwidth, load resistance, elementary charge, and average RIN spectral density. The photodiode dark current of the detector is small compared to the photocurrent and therefore is ignored. The values $R=0.4 \mathrm{~A} / \mathrm{W}, T=$ $298 \mathrm{~K}, F_{n}=5 \mathrm{~dB}, R_{L}=50 \Omega$, and $R I N=-155 \mathrm{~dB} / \mathrm{Hz}$ characterize our experimental setup and are used to find the theoretical BER for our experiments. The bandwidth was set to $12.5 \mathrm{GHz}$ for $12.5 \mathrm{Gbps} \mathrm{OOK}$ and to $12.5 \mathrm{GHz}$ for $25 \mathrm{Gbps} 4$-PAM and $6.25 \mathrm{GHz}$ for $12.5 \mathrm{Gbps} 4$-PAM. The symbols were set equispaced, and the thresholds were set in the middle between adjacent symbols. This would be optimal at high SNR if thermal noise was dominating and suboptimal if power-dependent noise (shot noise and RIN) was dominating.

The theoretical BER values for OOK and 4-PAM, both exact, obtained using Eq. (5), and approximated, including both Gray and natural labeling, obtained using Eq. (7), are illustrated in Fig. 1. The relative sensitivities of 4-PAM and OOK signals at the same bit-rate and at the same symbol rate agree with results from Eq. (2) and Eq. (1). The difference between the exact and approximate BER is very small in the low-BER region and becomes apparent only for very high BER. On the other hand, operation of short-range optical links in that

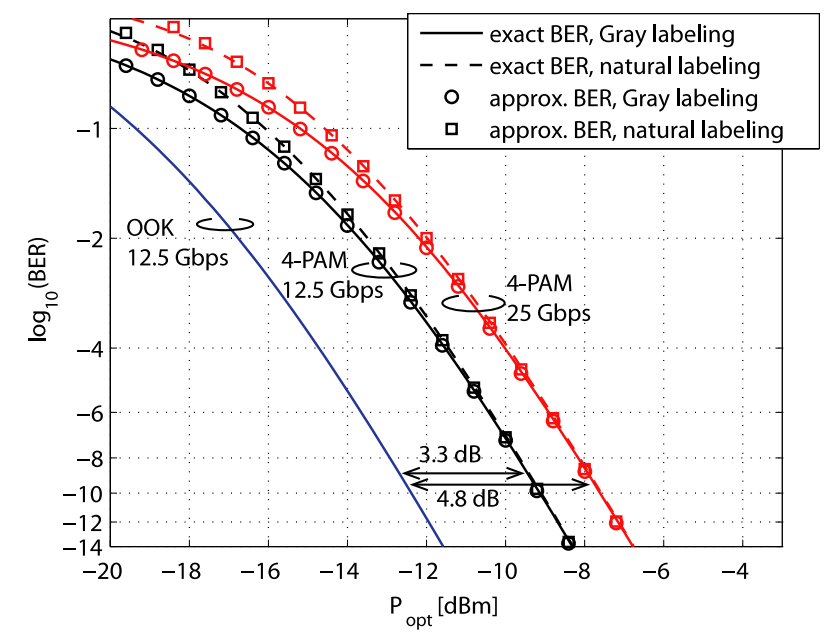

Fig. 1. (Color online) Theoretical BERs versus received optical power for OOK and 4-PAM. The approximate values of the BER were obtained using Eq. (7).

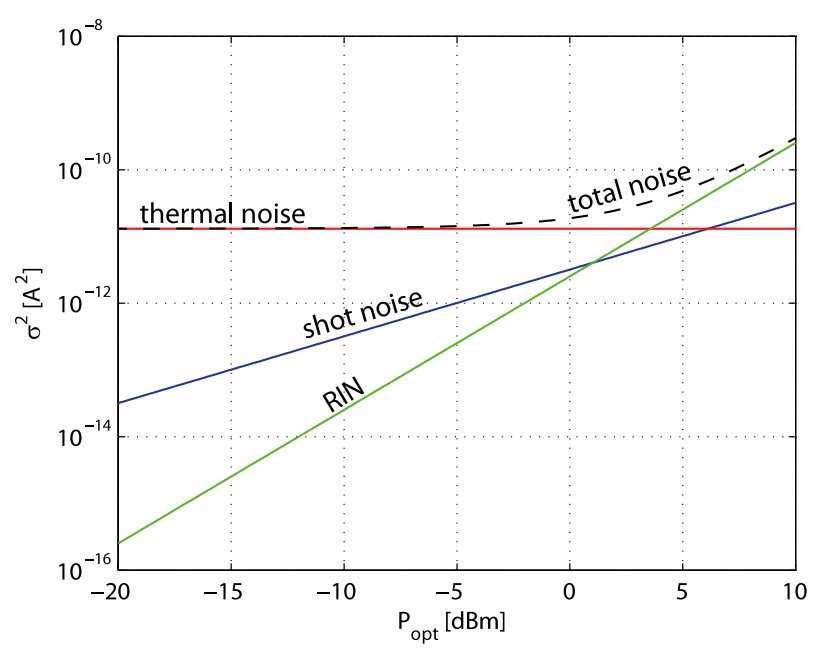

Fig. 2. (Color online) Contributions from the thermal noise, shot noise, and relative intensity noise for given system parameters at a receiver bandwidth of $12.5 \mathrm{GHz}$.

region is of limited interest in data center and supercomputing applications, because forward error correction (FEC) would have to be implemented. Although FEC would increase the power consumption and heat generation, and it is not used at present in short-range applications, it may become an option in the future. The small difference (at low BER) between the Gray and natural labelings is also worth noting. The penalty for using natural labeling is small, and it is easier to implement natural than Gray labeling in experiments.

The noise contributions are plotted in Fig. 2. The RIN and shot noise powers are stronger than RIN only for received power levels greater than $3 \mathrm{dBm}$. On the other hand, the photodetector at our disposal saturates at $3 \mathrm{dBm}$ received optical power, so the system is always dominated by the thermal noise. Since the thermal noise is not signal dependent, we can use equally spaced levels and decision thresholds at equal distances from the symbol levels $[9,18]$. 


\section{ISI Penalties Due to Modal Dispersion}

The ISI penalty due to the modal dispersion is well understood for OOK systems. The basic ISI penalty calculation methods have been outlined in [19]. The worst-case ISI penalty (expressed in $\mathrm{dB}$ ) is

$$
P_{\mathrm{ISI}}=10 \log _{10}\left(\frac{1}{1-E_{m}}\right)
$$

where $E_{m}$ is the worst-case eye closure. For OOK it is approximated as

$$
E_{m, \mathrm{OOK}}=1.425 \exp \left(-1.28\left(\frac{T}{T_{C}}\right)^{2}\right)
$$

The bit period is denoted as $T$ and the channel 10\%-90\% rise time is denoted as $T_{C}$. This ISI penalty calculation method, which is valid under assumptions of Gaussian channel response and rectangular input pulse, was given in [20]. It is used in the IEEE 802.3 link budget spreadsheet [21]. Methods of calculation of the $10 \%-90 \%$ rise time for a given system are described in [20].

We can now extend the ISI penalty estimates to 4-PAM, assuming that it contains three stacked OOK eye diagrams. Assuming that the channel response is Gaussian, it is easy to observe that, for the same system rise time and symbol rate, the eye closure for 4-PAM is twice as large as that for OOK,

$$
E_{m, 4 \mathrm{PAM}}=2.85 \exp \left(-1.28\left(\frac{T}{T_{C}}\right)^{2}\right) .
$$

It must be noted here that the top and bottom eye openings for 4-PAM signals are not symmetric, and therefore there is an additional power penalty if the decision thresholds are not adjusted.

The bandwidth of the experimental link was measured for various fiber lengths, and the results are presented in Subsection III.B. The measured system bandwidth was used to calculate the approximate theoretical ISI penalties for OOK and 4-PAM at $12.5 \mathrm{Gbps}$ and $25 \mathrm{Gbps}$ for both modulation formats. The theoretical ISI penalties are illustrated in Fig. 3. The theoretical ISI penalties for 4-PAM are substantially lower than for OOK at the same bit-rate, and at $25 \mathrm{Gbps}$ OOK suffers from ISI even in the back-to-back (BTB) case. The theoretical ISI penalties will be compared with experimental results in Section IV.

\section{EXPERIMENTAL SETUP}

\section{A. 4-PAM and OOK Signal Generation}

The OOK test signal was generated using an SHF 12103A pattern generator. The 4-PAM signal was generated from two binary pseudo-random binary signals (PRBS) of length $2^{7}-1$, at the same symbol rate as the target 4-PAM signal. It would be interesting to investigate the effects of longer PRBS patterns, since both VCSEL performance and effects of ISI are affected.

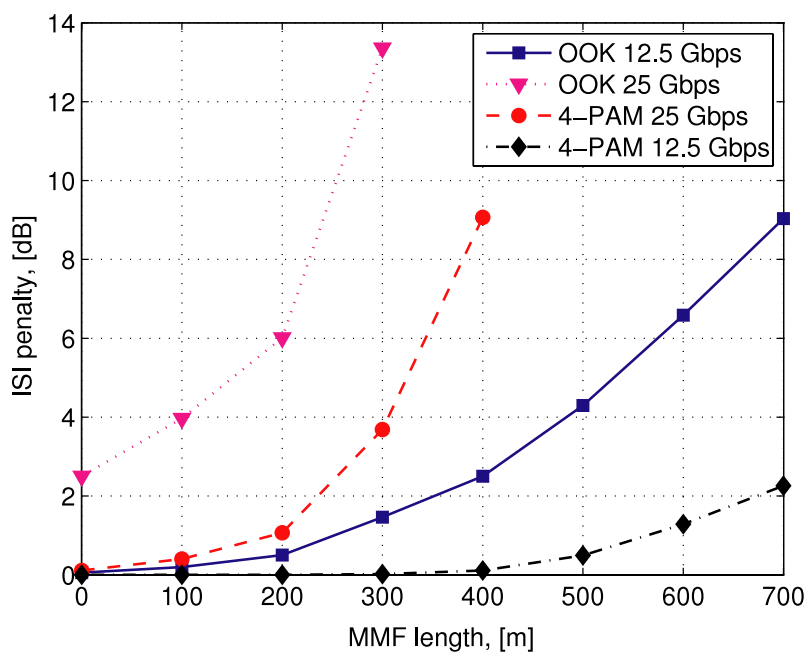

Fig. 3. (Color online) Theoretical ISI power penalties for OOK and 4-PAM.

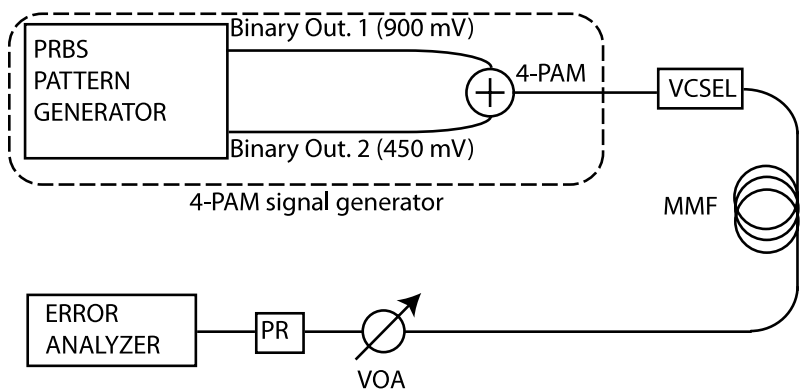

Fig. 4. Generalized experimental setup with 4-PAM signal generator. The photoreceiver (PR) and error analyzers were different in various configurations, which are listed in Table III.

On the other hand, short PRBS patterns are commonly used in experiments with VCSELs to model short run length codes, such as the $8 \mathrm{~B} 10 \mathrm{~B}$ code used in data communication applications [22,23]. The binary streams were offset by half of the pattern length for optimum decorrelation, which is important in order to obtain all transitions between the 4-PAM symbols and for proper SER measurement. It was also verified that the 4-PAM signal was bias neutral, i.e., it contained the same number of low-power and high-power symbols. The bits were mapped to symbols using natural coding, rather than Gray coding, which is an inherent characteristic of this way of generating a 4-PAM signal. The amplitudes of the binary signals were approximately $900 \mathrm{mV}$ and $450 \mathrm{mV}$, but they were adjusted slightly to optimize the resulting 4-PAM signal level spacing. The two binary signals were clock aligned and combined together, as illustrated in Fig. 4. The generated 4-PAM signal was then fed to a VCSEL through a bias-T.

\section{B. VCSEL and Transmission Fiber}

The VCSEL used in these experiments was developed in-house and comes from the same batch as the one reported in [24]. The VCSEL was biased at $8 \mathrm{~mA}$; at this bias current, 


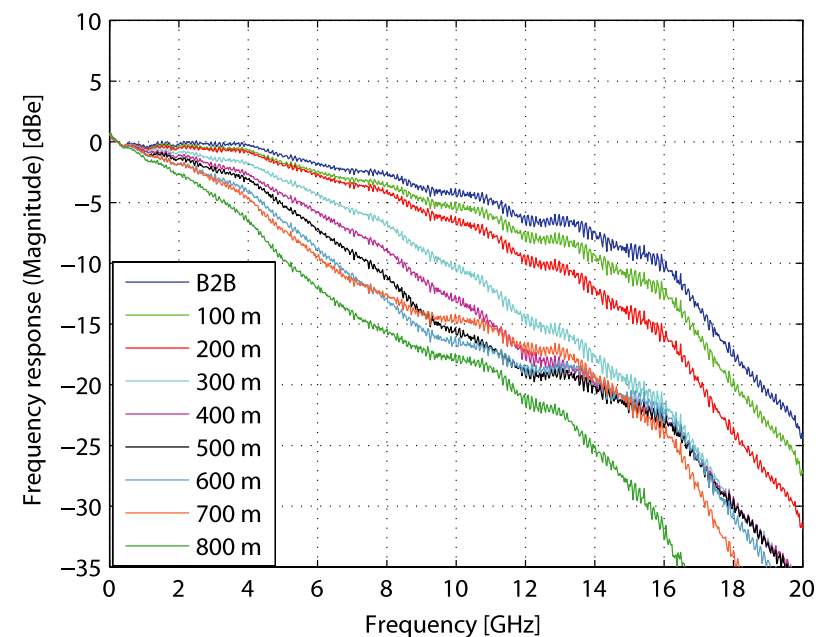

Fig. 5. (Color online) Normalized magnitude of the frequency response of the complete link for different fiber lengths.

the modulation bandwidth is around $16 \mathrm{GHz}$. Low bias current translates to low current density, which in turns improves reliability [9]. The VCSEL was operated without cooling, at room temperature. A photoreceiver with $12 \mathrm{GHz}$ bandwidth was used to detect the optical signal. Between the VCSEL and the photoreceiver, OM3+ graded index MMF was used, with a bandwidth-distance product of $4700 \mathrm{MHz} \cdot \mathrm{km}$. A variable optical attenuator was used to vary the optical power. The bandwidth of the MMF link was measured using a vector network analyzer. Fiber lengths up to $800 \mathrm{~m}$ were evaluated, with an increment of $100 \mathrm{~m}$. A BTB configuration was also included. The magnitude and phase of the frequency response are presented in Figs. 5 and 6, respectively. The bandwidths of the tested configurations are summarized in Table II. As the length of the MMF was increased, the bandwidth fell and roll-off increased. In the case of Gaussian frequency responses, the total bandwidth of a system can be expressed with a simple expression,

$$
\mathrm{BW}_{\text {system }}^{-2}=\mathrm{BW}_{1}^{-2}+\mathrm{BW}_{2}^{-2}+\mathrm{BW}_{3}^{-2}+\cdots,
$$

where $\mathrm{BW}_{\text {system }}$ denotes the total system bandwidth and $\mathrm{BW}_{i}$ denotes the component bandwidths [25]. It is easy to observe that in this case the total bandwidth of the system is less than the bandwidth of the slowest component. Even though real systems may have non-Gaussian frequency responses, Eq. (14) gives an illustration of the evolution of the system frequency response. The magnitude of the frequency response did not have any sharp dips or peaks, as would be expected for older types of MMF [26].

In many of the 4-PAM experiments reported, the modulation amplitude of the signal driving the VCSEL is adjusted to stay in the linear region of the static output power to driving current (PI) relationship. However, the semiconductor lasers have much more linear performance for high-frequency signals [27], and therefore static PI characteristics cannot be used to define linear operation. The static PI characteristics, as well as the static bias voltage to bias current characteristics, are plotted in Fig. 7. We have optimized experimentally the
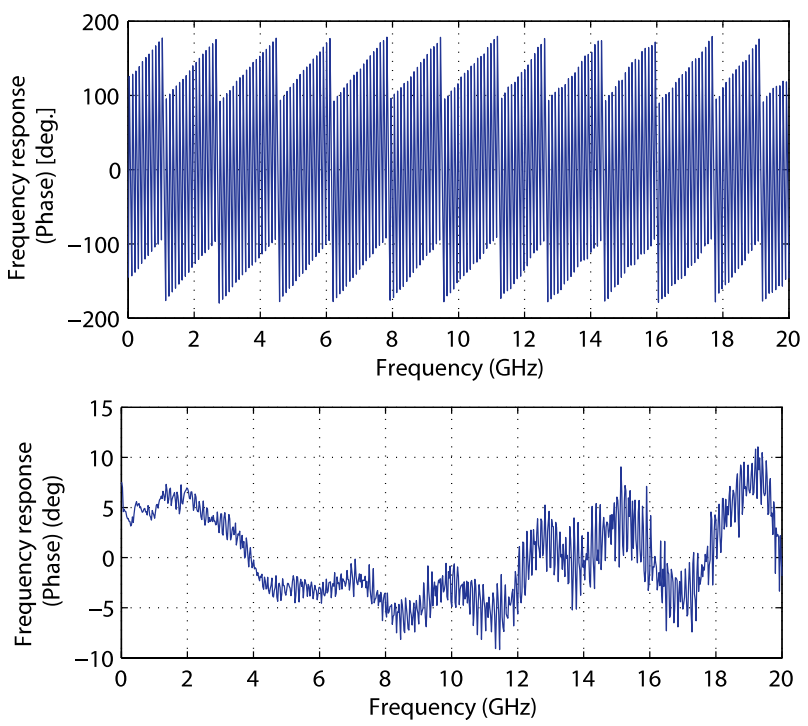

Fig. 6. (Color online) Phase response $\theta(f)$ of a system with $300 \mathrm{~m}$ of MMF (top plot) and the same phase response unwrapped and with the linear part subtracted, i.e., $\theta(f)-T f$, where $T=-62 \mathrm{~ns}$ (bottom plot).

TABLE II

MeAsured -3 dB BANDwidTh, -6 dB BANDWIDTH AND FREQUENCY ROLL-OFF FOR THE TESTED FIBER LINKS

\begin{tabular}{llll}
\hline Length $(\mathrm{m})$ & $\begin{array}{l}-3 \mathrm{~dB} \text { BW } \\
(\mathrm{GHz})\end{array}$ & $\begin{array}{l}-6 \mathrm{~dB} \mathrm{BW} \\
(\mathrm{GHz})\end{array}$ & $\begin{array}{l}\text { Roll-off } \\
(\mathrm{dB} / \text { decade })\end{array}$ \\
\hline 0 & 8.5 & 12 & 15 \\
\hline 100 & 7.2 & 11 & 16 \\
\hline 200 & 6.5 & 9.5 & 18 \\
\hline 300 & 5 & 7.5 & 22 \\
\hline 400 & 4.2 & 6 & 23 \\
\hline 500 & 4 & 5.5 & 24 \\
\hline 600 & 3.2 & 5 & 25 \\
\hline 700 & 3 & 4.5 & 26.5 \\
\hline 800 & 2.3 & 3.8 & 27.7 \\
\hline
\end{tabular}

driving signal to obtain the best signal. An example of a BTB 4-PAM eye diagram is presented in Fig. 8, to show that the eye diagrams are free from nonlinear distortions.

\section{Receiver Configurations}

At the time when the experiments were done, we had at our disposal a $12 \mathrm{GHz}$ photoreceiver with an integrated transimpedance amplifier (TIA). The responsivity of the photodiode in the photoreceiver was $0.4 \mathrm{~A} / \mathrm{W}$ at $850 \mathrm{~nm}$. For the error detection, we had two error analyzers (EAs): an Agilent N4903A and an SHF 11100B. The Agilent EA was used for signals with a symbol rate up to 12.5 Gbaud, and the SHF EA was used for signals with a symbol rate of 12.5 Gbaud and above. The photodetectors and EAs were used in the two configurations summarized in Table III.

The two configurations differ only with the choice of the EA. The SHF EA had better sensitivity, but the Agilent 


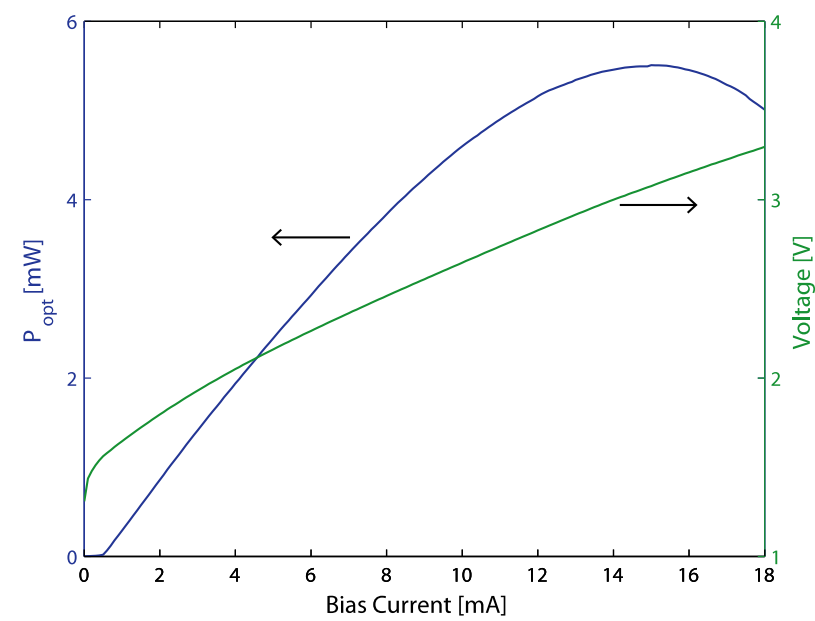

Fig. 7. (Color online) The power and voltage versus current characteristics of the VCSEL used in the experiments.

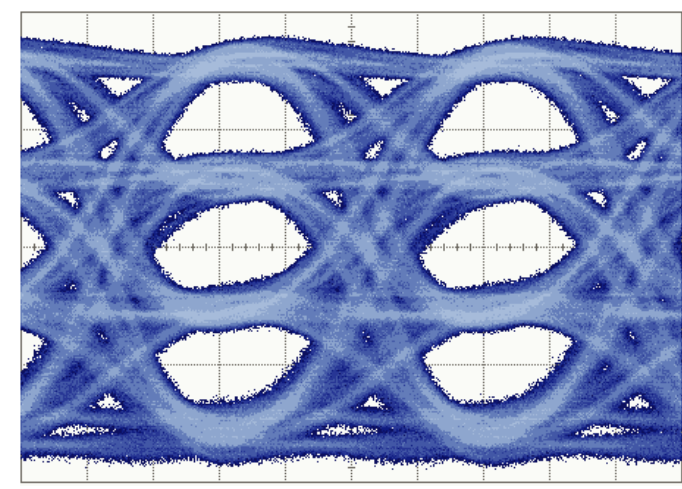

Fig. 8. (Color online) Back-to-back eye diagram of a 4-PAM signal at $25 \mathrm{Gbps}$, at $0 \mathrm{dBm}$ received optical power. Because of the inverting amplifier in the photoreceiver, the highest power level is in the bottom of the eye diagram.

TABLE III

RECEIVER CONFIGURATIONS

\begin{tabular}{llll}
\hline No. & Photodetector & Amplifier & Error analyzer \\
\hline 1 & $12 \mathrm{GHz}$ PR & Integrated & Agilent N4903A \\
\hline 2 & $12 \mathrm{GHz}$ PR & Integrated & SHF 11100B \\
\hline
\end{tabular}

EA could be used at lower symbol rates. Additionally, for the 12.5 Gbps 4-PAM signal, a $7.5 \mathrm{GHz}$ passive electrical low-pass filter (LPF) was added to reject the noise outside the signal bandwidth. Because of different performances of the two receiver configurations, all comparisons are always done with the same configuration. The test setup illustrated in Fig. 4 corresponds to the configuration with receiver no. 2 .

\section{Bit Error Rate Measurement}

The BER measurement was performed using an EA designed for conventional OOK modulation. Since it is the
low-BER operation that is of highest interest, it is enough to measure the error rates between the adjacent symbols (as motivated in Section II). This kind of measurement can be easily done with an EA with adequate decision threshold, programed with patterns corresponding to given decision thresholds. For 4-PAM, three decision thresholds are applicable. The error rates measured at these thresholds are denoted $\mathrm{ER}_{1}, \mathrm{ER}_{2}$, and $\mathrm{ER}_{3}$, and the total $\mathrm{BER}$ is given by

$$
\mathrm{BER}=\frac{1}{2} \mathrm{ER}_{1}+\mathrm{ER}_{2}+\frac{1}{2} \mathrm{ER}_{3},
$$

as was discussed in more detail in [14].

\section{RESUlTS AND Discussion}

\section{A. Comparison at the Same Symbol Rate-12.5 Gbaud}

The comparison of 4-PAM with OOK at the same symbol rate of 12.5 Gbaud was performed using receiver configuration no. 2. A BTB eye diagram at $0 \mathrm{dBm}$ received optical power is illustrated in Fig. 8: all levels had the same width, indicating that the noise is not signal dependent and that the theoretical considerations on noise in Section II are correct. The BER results are illustrated in Figs. 9 and 10. The BTB sensitivity at $\mathrm{BER}=10^{-9}$ of $\mathrm{OOK}$ at $12.5 \mathrm{Gbps}$ was about $-13 \mathrm{dBm}$, which agrees very well with the theory (see Fig. 1). On the other hand, 4-PAM at 25 Gbps had BTB sensitivity of around $-6.5 \mathrm{dBm}$, which was about $1.2 \mathrm{~dB}$ off the theoretical expectation, and consequently the difference between OOK and 4-PAM sensitivity was $6 \mathrm{~dB}$, rather than the expected $4.8 \mathrm{~dB}$.

The comparison of the receiver sensitivities versus propagation distance for the 12.5 Gbaud case is shown in Fig. 11. The propagation penalty for $200 \mathrm{~m}$ of the OM3+ MMF was small for both modulation formats, but, beyond this length, 4-PAM degraded much faster than OOK. This agrees with the theoretical ISI penalty evolution from Fig. 3. The absolute ISI penalties with respect to the BTB configuration are in good agreement with theory up to $400 \mathrm{~m}$ for OOK and up to $200 \mathrm{~m}$ for 4 -PAM, or roughly up to $2 \mathrm{~dB}$ ISI penalty, which comes at shorter distances for 4-PAM. Consequently, the approximate theoretical ISI expression for 4-PAM is useful for shorter fiber lengths. The theoretical ISI penalty is apparently overestimated for OOK and underestimated for 4-PAM for longer fiber lengths. The theoretical ISI penalties were calculated under the assumption of Gaussian channel response and pulse shape, which is only a rough approximation of the real conditions. For 4-PAM, the eye closing is larger for the two outer levels than in the center (see the insert in Fig. 11), while the theoretical model approximates it with the same eye closing for all levels.

\section{B. Comparisons at the Same Bit-Rate-12.5 Gbps}

The comparison at the same bit-rate was done at $12.5 \mathrm{Gbps}$. Receiver configuration no. 1 was used both for 4-PAM and OOK at $12.5 \mathrm{Gbps}$, with a $7.5 \mathrm{GHz}$ LPF for the 4-PAM. The BTB 


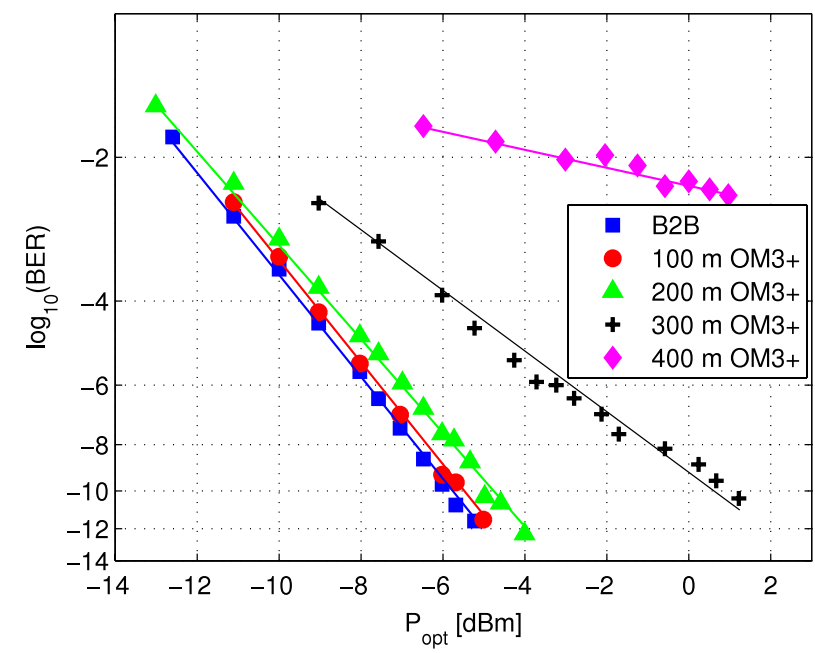

Fig. 9. (Color online) BER versus average received optical power for 4-PAM at $25 \mathrm{Gbps}$.

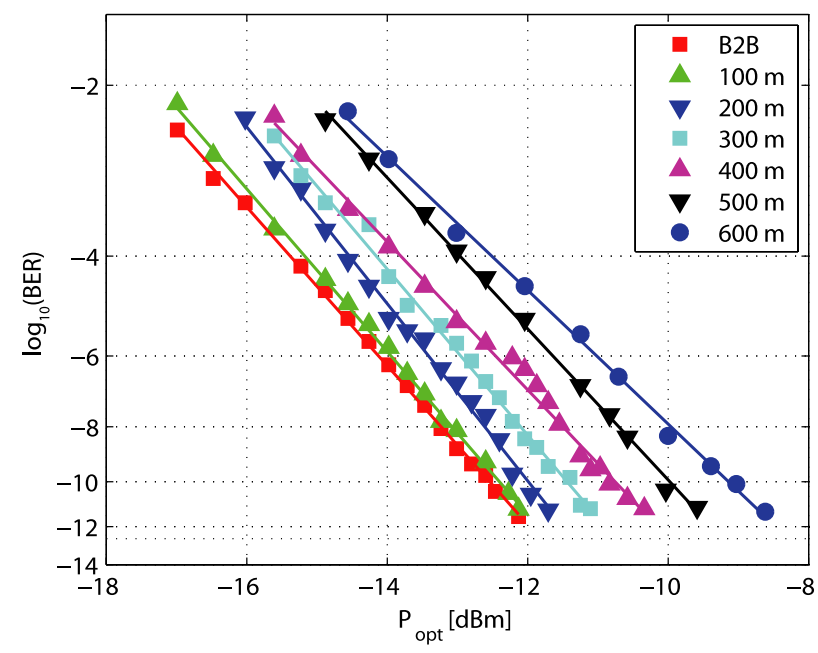

Fig. 10. (Color online) BER versus average received optical power for OOK at $12.5 \mathrm{Gbps}$, obtained with receiver in configuration no. 2.

receiver sensitivity of OOK with this receiver configuration was $4.5 \mathrm{~dB}$ worse than with receiver configuration no. 2 . The relative BTB sensitivity of 4-PAM at 12.5 Gbps was $4 \mathrm{~dB}$ worse than of OOK at $12.5 \mathrm{Gbps}$, while a $3.3 \mathrm{~dB}$ difference was expected from theory in Section II. The BER results for the 12.5 Gbps case are illustrated in Figs. 12 and 13. The receiver sensitivities after propagation over MMF are illustrated in Fig. 14. Within the available received power, both OOK and 4-PAM could reach $600 \mathrm{~m}$ at $12.5 \mathrm{Gbps}$, although the propagation penalty for 4-PAM is much lower until $500 \mathrm{~m}$ is reached. In fact, at $500 \mathrm{~m}$, the sensitivity of 12.5 Gbps 4-PAM was only $2.5 \mathrm{~dB}$ worse than of $12.5 \mathrm{Gbps}$ OOK. Beyond $500 \mathrm{~m}$, the propagation penalty for 4-PAM started to increase much faster. Similar behavior is observed in Fig. 3; however, the expected ISI penalty beyond $500 \mathrm{~m}$ is lower that what was observed in the experiments. From the theoretical consideration in Section II, it follows that

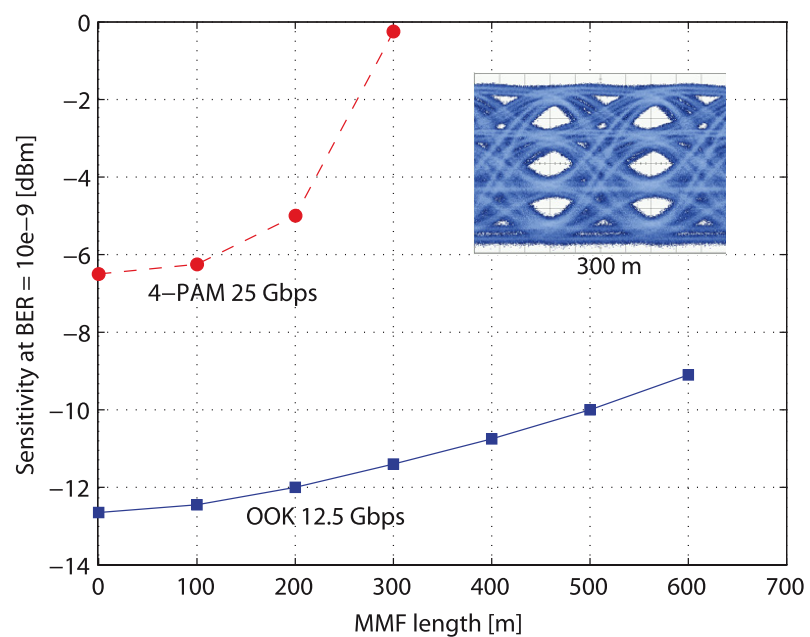

Fig. 11. (Color online) Sensitivity at BER $=10^{-9}$ versus MMF propagation distance compared at the same symbol rate (12.5 Gbaud) with 4-PAM eye diagram after $300 \mathrm{~m}$ in the insert.

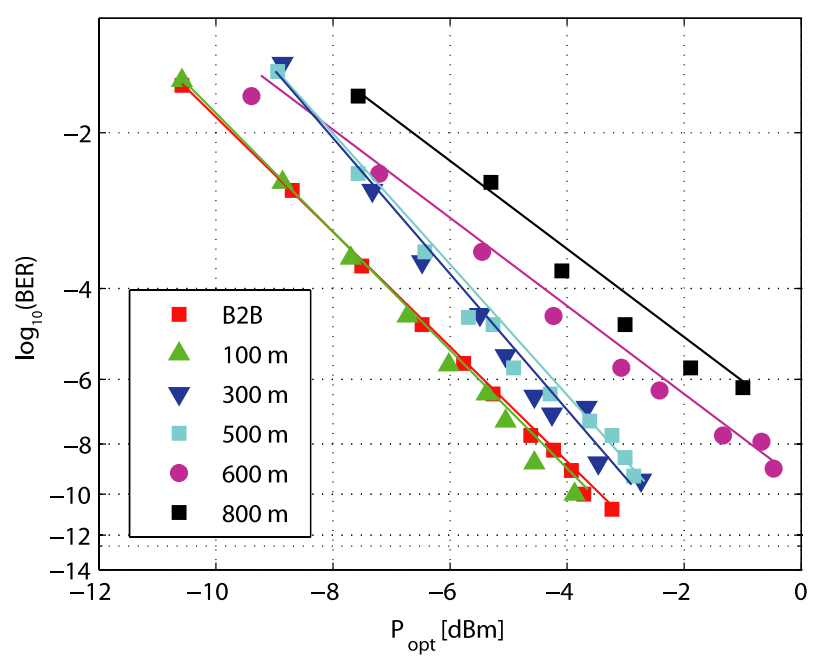

Fig. 12. (Color online) BER versus average received optical power for 4-PAM at $12.5 \mathrm{Gbps}$ and $25 \mathrm{Gbps}$.

4-PAM should have better sensitivity than OOK at the same bit-rate after propagation over $500 \mathrm{~m}$. In the experiments, the sensitivity and ISI penalties for 4-PAM were worse than the theoretical values. The theoretical penalties for 4-PAM are again underestimated compared to the experimental results. Similarly to the 12.5 Gbaud 4-PAM case, the eye closing becomes uneven for different levels, and that adds to the penalty, as illustrated in the insert in Fig. 14. There are also implementation penalties for 4-PAM, which could lower if electronic circuits dedicated for 4-PAM were developed. For example, the microwave combiners used are non-ideal, and a dedicated multilevel circuit would improve the performance. A highly linear optical receiver with automatic gain control (AGC), similar to the one used in [28], would make the BER measurements more reliable and simplify the design of a decision stage for 4-PAM in real applications. 


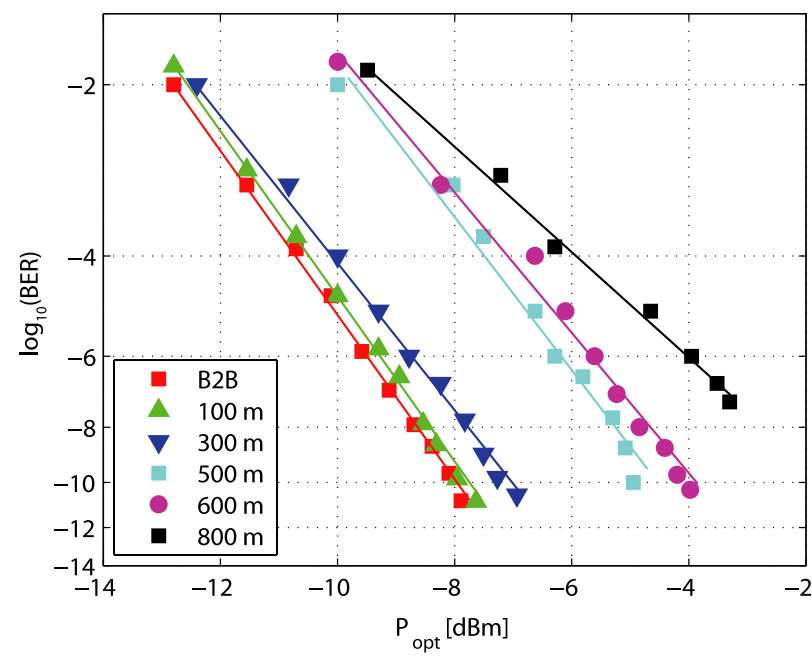

Fig. 13. (Color online) BER versus average received optical power for OOK at 12.5 Gbps, obtained with receiver in configuration no. 1.

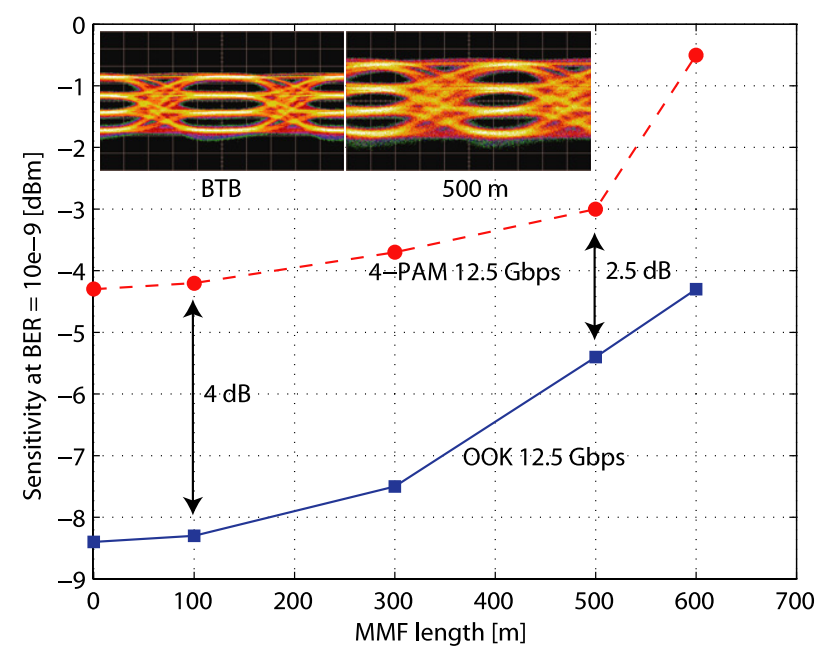

Fig. 14. (Color online) Sensitivity versus MMF propagation distance compared at the same bit-rate $(12.5 \mathrm{Gbps})$ with 4-PAM eye diagrams, $\mathrm{BTB}$ ad $500 \mathrm{~m}$, in the insert.

\section{Discussion}

The experiments show that 4-PAM required significantly more receiver power than OOK in both scenarios: $6 \mathrm{~dB}$ at the same symbol rate and $4 \mathrm{~dB}$ more at the same bit-rate. This is more than is expected from the theoretical considerations, which yield $4.8 \mathrm{~dB}$ and $3.3 \mathrm{~dB}$ in the two respective scenarios (Section II). The implementation penalties are thus $1.2 \mathrm{~dB}$ and $0.7 \mathrm{~dB}$, respectively. Possible reasons for the implementation penalties are the suboptimal electrical signal from the microwave couplers and lack of AGC in the receiver.

The ISI penalties of 4-PAM were lower than of OOK at the same bit-rate, but because of the implementation penalties we could not reach the point where the lower ISI penalties of 4-PAM would offset its higher power requirements. Also, the theoretical ISI penalties of 4-PAM were underestimated by 1-2 $\mathrm{dB}$ at longer fiber length, compared to the experimental results. This is reasonable, given the simplicity of the theoretical model, which assumes Gaussian pulses as well as channel responses and equal eye closing for all levels. The theoretical calculation gives good estimates up to roughly $2 \mathrm{~dB}$ of ISI penalty.

The difference in sensitivity between 4-PAM and OOK was comparable to, for example, the link budget foreseen for short data links in the IEEE 802.3 standard [29]. The link budget foreseen by this standard is $8.3 \mathrm{~dB}$ and allocation for penalties is $6.5 \mathrm{~dB}$. This means that, should an existing OOK system be upgraded to 4-PAM in order to double the bit-rate in the same bandwidth, the power budget would be exhausted. On the other hand, the power budgets might be different in applications such as active optical cables, where, for example, the connector losses are eliminated. Because of the closed nature of active optical cables, multi-source agreements do not have to be followed and, for example, the link budgets can be adjusted to the specific needs and 4-PAM could be used. As we show in our experiments, it is possible to make links with sufficient power budget for 4-PAM operation. The main benefit of 4-PAM in such an application would be doubled throughput, using the same components. In a bandwidth-constrained case, 4-PAM allows doubling the bit-rate and has lower propagation penalties, but the lower penalties after propagation in the MMF do not offset the higher optical power required by 4-PAM.

Given that at $12.5 \mathrm{Gbps}$ we need $-12 \mathrm{dBm}$ to achieve error-free performance with $\mathrm{OOK}$ and that the maximum available power at the photoreceiver is around $1 \mathrm{dBm}$, it should be possible to increase the number of modulation levels to 8 , since 8-PAM should require around $8.5 \mathrm{~dB}$ more power to reach the same BER (plus implementation penalty between 1 and $2 \mathrm{~dB})$.

\section{Conclusion}

We have shown that 4-PAM can be used to double the bit-rate of short-range optical links using existing components and $\mathrm{OOK}$, although it required $6 \mathrm{~dB}$ more optical power. We have also demonstrated that 4-PAM had lower propagation penalties than OOK at the same bit-rate, but required $4 \mathrm{~dB}$ more optical power in the BTB case. Due to implementation penalties, this was more than expected from the theoretical considerations. Because of the additional penalties, the reduced propagation penalties did not offset the increased power requirements of 4-PAM in the case of the particular tested system. Higher linearity requirements of 4-PAM, compared to OOK, were not a problem when VCSELs were used. Implementation of 4-PAM was relatively simple, and for experimental purposes does not require customized electronics.

\section{ACKNOWLEDGMENTS}

This work was supported by the Swedish Foundation for Strategic Research (SSF) and the European FP7 project VISIT, and we would like to thank them for their support. 


\section{REFERENCES}

[1] P. Westbergh, J. S. Gustavsson, B. Kögel, Å. Haglund, A. Larsson, A. Mutig, A. Nadtochiy, D. Bimberg, and A. Joel, "40 Gbit/s error-free operation of oxide-confined $850 \mathrm{~nm}$ VCSEL," Electron. Lett., vol. 46, no. 14, pp. 1014-1016, July 2010.

[2] W. Hofmann, P. Moser, P. Wolf, A. Mutig, M. Kroh, and D. Bimberg, "44 Gb/s VCSEL for optical interconnects," in Proc. OFC, Mar. 2011, PDPC5.

[3] K. Szczerba, B.-E. Olsson, P. Westbergh, A. Rhodin, J. S. Gustavsson, Å. Haglund, M. Karlsson, A. Larsson, and P. A. Andrekson, "37 Gbps transmission over $200 \mathrm{~m}$ of MMF using single cycle subcarrier modulation and a VCSEL with $20 \mathrm{GHz}$ modulation bandwidth," in Proc. ECOC, Sept. 2010, We.7.B.2.

[4] S. C. J. Lee, F. Breyer, S. Randel, D. Cardenas, H. P. A. van den Boom, and A. M. J. Koonen, "Discrete multitone modulation for high-speed data transmission over multimode fibers using 850-nm VCSEL," in Proc. OFC, Mar. 2009, OWM2.

[5] S. Randel, F. Breyer, and S. C. J. Lee, "High-speed transmission over multimode optical fibers," in Proc. OFC, Feb. 2008, OWR2.

[6] J. R. Kahn and J. M. Barry, "Wireless infrared communications," Proc. IEEE, vol. 85, no. 2, pp. 265-298, Feb. 1997.

[7] D. Watanabe, A. Ono, and T. Okayasu, "CMOS optical 4-PAM VCSEL driver with modal-dispersion equalizer for $10 \mathrm{~Gb} / \mathrm{s} 500 \mathrm{~m}$ MMF transmission," in Proc. ISSCC, Feb. 2009, pp. 106-107.

[8] T. Toifl, C. Menolf, M. Ruegg, R. Reutemann, P. Buchmann, M. Kossel, T. Morf, J. Weiss, and M. L. Schmatz, "A $22 \mathrm{~Gb} / \mathrm{s}$ PAM-4 receiver in 90-nm CMOS SOI technology," IEEE J. Solid-State Circuits, vol. 41, no. 4, pp. 954-965, Apr. 2006.

[9] S. Walklin and J. Conradi, "Multilevel signaling for increasing the reach of $10 \mathrm{~Gb} / \mathrm{s}$ lightwave systems," J. Lightwave Technol., vol. 17, no. 11, pp. 2235-2248, Nov. 1999.

[10] J. D. Ingham, R. V. Penty, and I. H. White, "10 Gb/s \& $20 \mathrm{~Gb} / \mathrm{s}$ extended-reach multimode-fiber datacommunication links using multilevel modulation and transmitter-based equalization," in Proc. OFC, Feb. 2008, OTuO7.

[11] J. D. Ingham, R. V. Penty, I. H. White, P. Westbergh, J. S. Gustavsson, A. Haglund, and A. Larsson, " 32 Gb/s multilevel modulation of an $850 \mathrm{~nm}$ VCSEL for next-generation datacommunication standards," in Proc. CLEO, May 2011, CWJ2.

[12] J. E. Cunningham, D. Beckman, X. Zheng, D. Huang, T. Sze, and A. V. Krishnamoorthy, "PAM-4 signaling over VCSELs with $0.13 \mu \mathrm{m}$ CMOS chip technology," Opt. Express, vol. 14, no. 25, pp. 12028-12038, Dec. 2006.

[13] K. Szczerba, P. Westbergh, J. S. Gustavsson, A. Haglund, J. Karout, M. Karlsson, P. A. Andrekson, E. Agrell, and A. Larsson, "30 Gbps 4-PAM transmission over $200 \mathrm{~m}$ of MMF using an 850 nm VCSEL," in Proc. ECOC, Sept. 2011, Tu.3.C.

[14] K. Szczerba, P. Westbergh, J. Karout, J. S. Gustavsson, A. Haglund, M. Karlsson, P. A. Andrekson, E. Agrell, and A. Larsson, "30 Gbps 4-PAM transmission over $200 \mathrm{~m}$ of MMF using an 850 nm VCSEL," Opt. Express, vol. 19, no. 26, pp. B203-B208, Dec. 2011.

[15] J. K. Pollard, "Multilevel data communication over optical fibre," IEE Proc.-Commun., vol. 138, no. 3, pp. 162-168, June 1991.

[16] J. G. Proakis and M. Salehi, Digital Communications. 5th ed. McGraw-Hill, New York, 2008.

[17] E. Agrell, J. Lassing, E. G. Ström, and T. Ottosson, "On the optimality of the binary reflected Gray code," IEEE Trans. Inf. Theory, vol. 50, no. 12, pp. 3170-3182, Dec. 2004.

[18] G. P. Agrawal, Lightwave Technology: Telecommunication Systems. Wiley Interscience, New York, 2005.

[19] J. Gimlett and N. Cheung, "Dispersion penalty analysis for LED/single-mode fiber transmission systems," J. Lightwave Technol., vol. 4, no. 9, pp. 1381-1392, Sept. 1986.
[20] D. Cunningham, M. Nowell, D. Hanson, and L. Kazovsky, The IEEE 802.3z Worst Case Link Model for Optical Physical Media Dependent Specification [Online]. Available: http://www.ieee802. org/3/z/public/presentations/mar1997/DCwpaper.pdf.

[21] IEEE 802.3ae 10G Ethernet optical link budget spreadsheet [Online]. Available: http://ieee802.org/3/10G_study/public/email_ attach/All_1250v2.xls.

[22] J. D. Ingham, R. V. Penty, and I. H. White, "Modulation formats for next-generation optical datacommunications," in Proc. ICTON, June 2011, Mo.C1.2.

[23] J. D. Ingham, R. V. Penty, I. H. White, and D. Cunningham, " $40 \mathrm{~Gb} / \mathrm{s}$ carrierless amplitude and phase modulation for low-cost optical datacommunication links," in Proc. OFC, Mar. 2011, OThZ3.

[24] P. Westbergh, J. S. Gustavsson, A. Haglund, A. Larsson, F. Hopfer, G. Fiol, D. Bimberg, and A. Joel, " 32 Gbit/s multimode fibre transmission using high-speed, low current density $850 \mathrm{~nm}$ VCSEL," Electron. Lett., vol. 45, no. 7, pp. 366-368, Mar. 2009.

[25] G. Brown, "Bandwidth and rise time calculations for digital multimode fiber-optic data links," J. Lightwave Technol., vol. 10, no. 5, pp. 672-678, May 1992.

[26] A. M. E.-A. Diab, J. D. Ingham, R. V. Penty, and I. H. White, "Statistical analysis of subcarrier-modulated transmission over $300 \mathrm{~m}$ of $62.5-\mu \mathrm{m}$-core-diameter multimode fiber," J. Lightwave Technol., vol. 23, no. 8, pp. 2380-2398, Aug. 2005.

[27] W. I. Way, Broadband Hybrid Fiber Coax Access System Technologies, 1st ed. Academic Press, Orlando, FL, 1998.

[28] M. Atef, R. Swoboda, and H. Zimmermann, “ $170 \mathrm{Mb} / \mathrm{s}$ multilevel transmission over $115 \mathrm{~m}$ standard step-index plastic optical fiber using an integrated optical receiver," Opt. Commun., vol. 284, no. 1, pp. 191-194, 2011.

[29] IEEE Standard for Information Technology-Telecommunications and Information Exchange Between Systems-Local and Metropolitan Area Networks-Specific Requirements, IEEE Std 802.3ba-2010, 2010.

Krzysztof Szczerba received his M.Sc. degree in Telecommunications from the Technical University of Denmark, Lyngby, Denmark, and his M.Sc. degree in Electronics and Telecommunications from the Technical University of Lodz, Poland, both in 2008. In 2008, he worked at the Technical University of Denmark, DTU Fotonik, Department of Photonics Engineering as a research assistant. In 2009, he joined the Chalmers University of Technology, Department of Microtechnology and Nanoscience, in Göteborg, Sweden, where he is currently working toward a $\mathrm{Ph} . \mathrm{D}$. degree in optical communications. His research interests are focused on modulation formats for short-range optical links for datacommunication applications.

Petter Westbergh received his M.Sc. degree in Engineering Physics and his Ph.D. degree in Microtechnology and Nanoscience from Chalmers University of Technology, Göteborg, Sweden, in 2007 and 2011, respectively. His thesis focused on the design, fabrication, and characterization of high-speed $850 \mathrm{~nm}$ vertical-cavity surface-emitting lasers (VCSELs) intended for application in short-reach communication networks. He is currently continuing his work on improving the performance of high-speed VCSELs with the Department of Microtechnology and Nanoscience at Chalmers University of Technology.

Johnny Karout received his B.E. (with distinction) in Computer Engineering from the Lebanese American University, Byblos, Lebanon, in 2007, and his M.Sc. in Communication Engineering and Licentiate degree in Electrical Engineering, both from Chalmers University of Technology, Göteborg, Sweden, in 2009 and 2011, respectively. Since June 2009, he has been pursuing his Ph.D. at the Department of Signals and Systems, Chalmers University of Technology, Göteborg, Sweden. 
From October to December 2011, he was a visiting scholar at the Institute for Communications Engineering at the Technische Universität München, Germany. His research interests are in the broad areas of modulation and coding, with a focus on optical channels.

$\mathrm{He}$ is the co-founder of the project Optium, which is related to short-haul optical communications at Chalmers Innovation, a business incubator located in Göteborg, Sweden. He is also a recipient of the 2011 GlobeCom Best Paper Award.

Johan S. Gustavsson received his M.Sc. degree in Electrical Engineering and his Ph.D. degree in Photonics from the Chalmers University of Technology, Göteborg, Sweden, in 1998 and 2003, respectively.

He was involved in mode dynamics and noise in vertical-cavity surface-emitting lasers (VCSELs). He is currently an Associate Professor in the Photonics Laboratory, Department of Microtechnology and Nanoscience, Chalmers University of Technology. His current research interests include the design, modeling, and characterization of long-wavelength semiconductor lasers, mode and polarization control in VCSELs using shallow surface structures, and improving the bandwidth of conventional GaAs-based VCSELs.

Åsa Haglund received her M. Sc. degree in Physics from Göteborg University, Göteborg, Sweden, in 2000, and her Ph.D. degree in Electrical Engineering from Chalmers University of Technology, Göteborg, Sweden, in 2005. Her Ph.D. thesis was focused on mode and polarization control in vertical-cavity surface-emitting lasers (VCSELs) using surface structures. She is currently an Assistant Professor at the Photonics Laboratory at Chalmers University of Technology, and her research is mainly focused on GaAs-based verticalcavity surface-emitting lasers and III-nitride-based vertical-cavity surface-emitting lasers and deep-ultraviolet light-emitting diodes.

Magnus Karlsson received his Ph.D. in Electromagnetic Field Theory in 1994 from Chalmers University of Technology, Göteborg, Sweden. The title of his Ph.D. thesis was "Nonlinear propagation of optical pulses and beams." Since 1995, he has been with the Photonics Laboratory at Chalmers, first as an Assistant Professor and since 2003 as a Professor in Photonics. He has authored or co-authored over 190 scientific journal and conference contributions, served as guest editor for the Journal of Lightwave Technology, and is currently an associate editor of Optics Express. He has served in the technical committees for the Optical Fiber Communication Conference (OFC) (2009 as subcommittee chair), and the Asia Communications and Photonics Conference (ACP, formerly APOC). His research has been devoted to a variety of aspects of fiber optic communication systems, in particular transmission effects such as fiber nonlinearities and polarization effects, but also applied issues such as high-capacity data transmission and all-optical switching. Currently he is devoted to parametric amplification, multilevel modulation formats, and coherent transmission in optical fibers.

Peter Andrekson received his Ph.D. from Chalmers University of Technology, Sweden, in 1988. After about three years with AT\&T Bell Laboratories, Murray Hill, NJ, USA, during 1989-1992, he returned to Chalmers, where he is now a Full Professor in the Department of Microtechnology and Nanoscience. He was Director of Research at Cenix Inc. in Allentown, PA, USA, during 2000-2003 and with the newly established Center for Optical Technologies at Lehigh University, Bethlehem, PA, USA, during 2003-2004. His research interests include nearly all aspects of fiber communications such as optical amplifiers, nonlinear pulse propagation, all-optical functionalities, and very high-capacity transmission. He is a co-founder of the optical test and measurement company Picosolve Inc., now part of EXFO; he is a Director of EXFO Sweden AB.

Andrekson is a Fellow of the Optical Society of America and a Fellow of the IEEE. He is the author of about 350 scientific publications and conference papers in the area of optical communications, among which 80 were invited papers at leading international conferences and journals, including two tutorials at the Optical Fiber Communication Conference (OFC) in 2004 and 2011. He is an elected member of the Board of Governors for the IEEE Photonics Society and is serving or has served on several technical program committees, including OFC and ECOC, and as an international project and candidate evaluator, and has also twice served as an expert for the evaluation of the Nobel Prize in Physics. He was an associate editor for IEEE Photonics Technology Letters during 2003-2007. In 1993, he was awarded a prize from the Swedish government research committee for outstanding work performed by young scientists, and in 2000 he was awarded the Telenor Nordic research award for his contribution to optical technologies.

Erik Agrell received his M.S. degree in Electrical Engineering in 1989 and his Ph.D. degree in Information Theory in 1997, both from Chalmers University of Technology, Sweden.

From 1988 to 1990 he was with Volvo Technical Development as a Systems Analyst, and from 1990 to 1997 he was with the Department of Information Theory, Chalmers University of Technology, as a Research Assistant. In 1997-1999, he was a Postdoctoral Researcher with the University of Illinois at Urbana-Champaign and the University of California, San Diego. In 1999, he joined the faculty of Chalmers University of Technology, first as an Associate Professor and since 2009 as a Professor in Communication Systems. His research interests belong to the fields of information theory, coding theory, and digital communications, and his favorite applications are found in optical communications. More specifically, his current research includes bit-interleaved coded modulation and multilevel coding, polarization-multiplexed coding and modulation, transmission over the optical intensity channel, phase noise mitigation, and bit-to-symbol mappings in coded and uncoded systems.

Anders Larsson received his M.Sc. and Ph.D. degrees in Electrical Engineering from Chalmers University of Technology, Göteborg, Sweden, in 1982 and 1987, respectively. In 1991, he joined the faculty at Chalmers University of Technology, where he was promoted to Professor in 1994. From 1984 to 1985 he was with the Department of Applied Physics, California Institute of Technology, and from 1988 to 1991 with the Jet Propulsion Laboratory, both at Pasadena, CA, USA. He has been a guest professor at Ulm University (Germany), at the Optical Science Center, University of Arizona, Tucson, Arizona (USA), at Osaka University (Japan), and at the Institute of Semiconductors, Chinese Academy of Sciences (China). He co-organized the IEEE Semiconductor Laser Workshop 2004, organized the European Semiconductor Laser Workshop 2004, and was the Program and General Chair for the IEEE International Semiconductor Laser Conference in 2006 and 2008, respectively. He is an associate editor for the IEEE Journal of Lightwave Technology. His scientific background is in the areas of materials and devices for optical communication, information processing, and sensing. Currently, his research is focused on vertical-cavity surface-emitting lasers, optically pumped semiconductor disk lasers, and III-nitride emitters. He has published more than 400 scientific journal and conference papers. He is a Fellow of the European Optical Society and a Senior Member of IEEE. 